\title{
Predictors of short- and long-term mortality among acutely admitted older patients: role of inflammation and frailty
}

\author{
Michela Zanetti ${ }^{1,2}$ (C) Giovanna Marzaro ${ }^{1} \cdot$ Paolo De Colle $^{2} \cdot$ Gabriele Toigo $^{1} \cdot$ Dario Bianchini $^{2} \cdot$ Mariapaola Nastri $^{1,2}$. \\ Cristina Suriano $^{1,2} \cdot$ Rocco Barazzoni $^{1,2} \cdot$ Gianfranco Sanson $^{1}$ (i)
}

Received: 24 April 2021 / Accepted: 24 June 2021 / Published online: 13 July 2021

(c) The Author(s) 2021

\begin{abstract}
Background Frailty, demographic and clinical variables linked to incident diseases (e.g., dehydration, inflammation) contribute to poor outcomes in older patients acutely hospitalized. Their predictivity on short-, intermediate- and long-term mortality in a comprehensive model has been scarcely investigated.

Aims To test the performance of a predictive tool considering frailty and inflammation as well as age, sex and impaired hydration status on 1-year mortality in acutely admitted older patients.

Methods Retrospective observational study including 529 medical patients (age 84.6 \pm 7.3 years). At hospital admission, frailty was assessed by the Multidimensional Prognostic Index (MPI). The Glasgow Prognostic Score (GPS) was used to grade systemic inflammation. Serum osmolarity was calculated to assess hydration.

Results After adjusting for age, sex, GPS and osmolarity, the severe-risk MPI was a strong predictor for 1-year mortality (OR 4.133; 95\% CI 2.273-7.516; $p<0.001$ ). Age $>85$ years, male sex, GPS-2 and serum osmolarity $>300 \mathrm{mOsm} / \mathrm{L}$ were independent predictors of mortality in the same multivariable model. The MPI alone showed a moderate discrimination power (AUC $0.678 ; 95 \%$ CI $0.628-0.729 ; p<0.001$ ) on 1-year mortality, which increased by $12.5 \%$ after the addition of the above predictors in the fully adjusted regression model (AUC $0.763 ; 95 \%$ CI $0.719-0.807 ; p<0.001$ ). The severe-risk MPI adjusted for the same factors was also an independent predictor of mortality after 60 and 180 days since hospital admission. Discussion Inflammation and impaired hydration are potentially modifiable risk factors for severe outcomes in older acutely hospitalized patients. A model combining GPS, age, gender, and plasma osmolarity improved the accuracy of MPI at admission in predicting long-term mortality.
\end{abstract}

Keywords Glasgow Prognostic Score · Inflammation · Multidimensional Prognostic Index · Osmolarity · Mortality · Hospital

\section{Introduction}

The need for hospital admission due to acute medical conditions increases with age as well as the rate of morbidity and mortality [1]. The risk of prolonged hospital stay, developing geriatric syndromes, institutionalization and death is higher in older frail patients [2] making diagnosis

Gianfranco Sanson

gsanson@units.it

1 Department of Medical, Surgical and Health Sciences, University of Trieste, Strada di Fiume 447, 34100 Trieste, Italy

2 Department of Internal Medicine, University Hospital, Trieste, Italy and scoring severity of frailty an essential requisite to guide decision making and treatment plan [3, 4]. The Multidimensional Prognostic Index (MPI), derived from the Comprehensive Geriatric Assessment (CGA), includes information on functional, nutritional, cognitive and social status, polypharmacy and comorbidities. The MPI has proved to represent an excellent instrument to predict short- and long-term adverse clinical outcomes including mortality in older frail patients affected by several diseases $[5,6]$.

In addition to frailty, a cluster of clinical and demographic factors including age and sex is associated with poor prognosis and mortality in elderly patients [7-12]. Although studies demonstrated an increased prognostic value of MPI according to sex and older age [13, 14], at present these factors are not included in this tool. Among clinical factors, 
systemic inflammation correlates with important clinical outcomes in older patients acutely hospitalized, including mortality, duration of hospital stay and nonelective readmissions [15]. Although increased plasma levels of cytokines, such as interleukin-6, have been correlated with short-term mortality in hospitalized older patient independently of frailty [16], their widespread use and applicability is limited by the availability of laboratory measurement. Therefore, alternative biomarkers easily obtainable in clinical practice should be identified and tested for mortality prediction. The Glasgow Prognostic Score (GPS), a simple tool derived from the combination of C-reactive protein and albumin levels [17], has been developed and validated to predict mortality in cancer patients and in conditions characterized by inflammation and/or malnutrition including COPD, relapse of idiopathic pulmonary fibrosis and myocardial infarction in the elderly [18-21].

Another useful assessment is that of hydration status, since dehydration is common in hospitalized older adults ranging from over one third to $50 \%$ of geriatric adults admitted to the hospital as emergencies [22, 23]. Impaired hydration is easily diagnosed by serum osmolality or alternatively by calculated serum osmolarity by validated formulas [24, 25]. Dehydration may develop quickly in those at increased risk with life-threatening consequences both in hospital after discharge [22, 26-28]. Rapid identification of this condition allows to establish a prompt treatment, which results in complete reversibility in mild and impending forms. Despite its high recurrence rates and poor prognosis, dehydration is often overlooked and it is not included within clinical algorithms developed for mortality prediction of older hospitalized patients.

Thus, the objectives of this study were: (1) to test the independent associations with short-, intermediate- and long-term mortality of inflammation, impaired hydration, and frailty adjusted for age and sex at hospital admission (2) to assess whether the addition of one or more variables independently affecting mortality can enhance the predictive performance of the MPI and its risk categories on 1-year mortality.

\section{Materials and methods}

\section{Study design, setting and population}

This was a retrospective observational study conducted in the Department of Medicine, Geriatric Unit, University Hospital of Trieste, Italy. This study was based on a secondary analysis of a prospectively collected database. All consecutive patients aged $\geq 65$ years and admitted from January 1st 2019 to December 31st 2019 from the Emergency Department were considered for eligibility. For patients presenting more than one admission into the Geriatric Unit, only the first admission during the study period was considered. Patients with incomplete CGA assessment were excluded.

A minimum required sample size of 442-448 patients was calculated to detect, with a probability of a type I error of 0.05 and a type II error of 0.2 , a 1-year mortality rate difference between MPI severe- and moderate-risk groups of approximately $30 \%$, according to previous literature [14].

\section{Ethics}

This study is part of the PROPOSING IDEAS (PROgnostic factors of Poor hospital Outcome based on clinical Severity, nutrItional and NursinG indexes IDEntified on AdmiSsion in elderly/frail patients) study, approved by the Regional Bioethics Committee of Friuli Venezia Giulia, Italy (Protocol number: 28217; date of approval: 31 July 2018). All the procedures performed in the study were in accordance with the Declaration of Helsinki and can be classified as "usual practice". At hospital admission, all enrolled patients authorised the use of their clinical data for study purposes.

\section{Study variables}

Clinical, functional and laboratory data assessed on admission were collected from the hospital electronic archive and from patients' medical records.

The MPI was computed upon hospital admission based on the routinely collected Comprehensive Geriatric Assessment (CGA), comprising data on number of drugs at admission, cohabitation status, functional (by Basal and Instrumental Activities of Daily Living), cognitive (by Short Portable Mental Status Questionnaire) and nutritional status (by Mini Nutritional Assessment), risk of developing pressure sores (by Exton-Smith Scale) and comorbidities (by the Cumulative Illness Rating Scale). The MPI was thus calculated by including the information from the above-reported domains of the CGA according to the methodology previously reported, establishing three grades of MPI (range: 0 to 1): low risk (0.0-0.33); moderate risk (0.34-0.66); and severe risk (0.67-1.0) [29].

Serum osmolarity was calculated based on the equation by Krahn and Khajuria [30], validated in very different populations of older adults [31] (osmolarity $=1.86 \times\left(\mathrm{Na}^{+}+\mathrm{K}^{+}\right)+1.15 \times$ glucose + urea +14 ; each component measured in $\mathrm{mmol} / \mathrm{L}$ ). Patients were considered dehydrated if calculated osmolarity was $>300 \mathrm{mmol} / \mathrm{L}$, while a condition of impending dehydration was defined with an osmolarity of $296-300 \mathrm{mmol} / \mathrm{L}$.

GPS was calculated based on the combination of C-reactive protein and albumin levels and the following categories were identified: 0 (good prognosis$\mathrm{CRP} \leq 10 \mathrm{mg} / \mathrm{L}$ and albumin $\geq 3.5 \mathrm{~g} / \mathrm{dL}$ ), 1 (intermediate 
prognosis-CRP $>10 \mathrm{mg} / \mathrm{L}$ and albumin $\geq 3.5 \mathrm{~g} / \mathrm{dL}$, or $\mathrm{CRP} \leq 10 \mathrm{mg} / \mathrm{L}$ and albumin $<3.5 \mathrm{~g} / \mathrm{dL}$ ) and 2 (poor prognosis-CRP $>10 \mathrm{mg} / \mathrm{L}$ and albumin $<3.5 \mathrm{~g} / \mathrm{dL}$ ).

\section{Study end-points}

The primary study endpoint was mortality—defined as death from any cause-within 12 months from hospital admission. Furthermore, hospital length of stay and all-cause mortality at 60 and 180 days were analyzed as secondary endpoints. Date of death was obtained from the public Regional electronic mortality register.

\section{Data analysis}

Data distribution was evaluated using the Kolmogorov-Smirnov test. The difference between the means was analyzed using the unpaired Student $t$-test, after determining whether equal variance could be attributed to the subgroups according to Levene's test. One-way analysis of variance (ANOVA) was applied for all comparisons between the subgroups. The nominal variables were described as a number and percentage, and analyzed with contingency tables and the $\chi$ test.

Five logistic regression models were tested to verify the extent of which the addition of covariates supposed to affect mortality independently from MPI (i.e.: age, sex, GPS, and serum osmolarity) affects the predictive power (that is, the ability to anticipate an outcome) of the MPI to predict 1-year patient mortality. Four categories (i.e.: $\leq 80 ; 81-85$; 86-90; and $>90$ years) were created according to 25 th, 50th and 75 th percentiles of the study population age. The following covariates were subsequently added to a simple logistic regression model (Model 1) consisting of MPI alone: age and sex (Model 2); age, sex and osmolarity (Model 3); age, sex and GPS (Model 4); and age, sex, GPS and osmolarity (Model 5). Results were reported as unadjusted an adjusted odds ratios (OR) with respective $95 \%$ confidence intervals (CI). The coefficient of the determination of each model was calculated based on the Nagelkerke $R^{2}$. The fitted values (predicted probability) from the multivariable regression models were used to construct receiver operating characteristic (ROC) curves and to compare this with the ROC curve derived from unadjusted MPI. The performance of the above predictive models in discriminating between patients who would die or not after 1 year from hospital admission was tested by calculating the area under ROC curve (AUC), which results were interpreted according to the following criteria: 0.50-0.59: poor; 0.60-0.69: moderate; $0.70-0.79$ : good; 0.80-0.89: very good; and $\geq 0.90$ : excellent discrimination [32]. Since the performance of the predictive model could have been overestimated as simply established on the sample used to build it, the possible 'over-optimism' in the performance of the final model was tested by the bootstrapping technique, as this analysis was recommended for estimating internal validity of predictive logistic regression models [33]. Three-thousand replications of random sampling with replacement by drawing same size samples (i.e., 529 subjects) from the original data set were performed. The difference between the AUC obtained by the bootstrapping re-sampling method and the original AUC represented the optimism for the study data set.

Forward stepwise multivariate logistic regression models were run to test the predictive power of the MPI on patient mortality at different time intervals, controlled for the above covariates. Multivariate Cox regression models with forward stepwise selection were used as a sensitivity analysis to estimate the time-to-event effect of the MPI categories on the risk of death at the same time intervals. Results were reported as unadjusted an adjusted hazard ratios (HR) with respective $95 \% \mathrm{CI}$ and adjusted survival curves.

All statistical analyses were performed using the software IBM SPSS Statistics, version 24.0 (Armonk, NY, US: IBM Corp.), while for the bootstrapping procedure a dedicated software was used [34]. For all tests, an alpha level of $p \leq 0.05$ was set for statistical significance.

\section{Results}

During the study period, 587 patients were admitted to the study ward. All patients had a complete MPI assessment on admission. After excluding subjects having more the one admission in the study period, 529 patients constituted the study population. For each, the complete outcome data were available. Table 1 shows the main characteristics of the study population. After excluding the patients who dead before hospital discharge, the hospital LOS for patients discharged alive from hospital $(n=526,99.4 \%)$ was $10.9 \pm 7.7$ days.

In the bivariate analysis, MPI was higher $(p<0.001)$ for patients who died within 1 year from hospital admission $(n=196 ; 0.60 \pm 0.20)$ compared to those who survived $(n=333 ; 0.44 \pm 0.20)$. Moreover, 1-year mortality was significantly related to higher GPS, osmolarity and age and male sex (Table 2). No statistically significant difference in hospital LOS was found for patients presenting a severe-risk MPI compared to low- or moderate-risk $(p=0.658)$, while the LOS was significantly longer for patients with higher GPS (GPS $<2: 9.1 \pm 6.9$ days; $\mathrm{GPS}=2: 12.8 \pm 8.4$ days; $p<0.001$ ) and osmolarity (osmolarity $\leq 300: 10.3 \pm 6.5$ days; osmolarity > 300: $12.2 \pm 10.0$ days; $p=0.043$ ).

The logistic regression models (Table 2 ) showed that only severe-risk MPI category maintains its power as a predictive tool on 1-year mortality in the fully adjusted final model. Moreover, the performance of severe-risk MPI category falls (OR from 6.2 to 4.1) when it was adjusted for the considered 
Table 1 Main characteristics of the study population

\begin{tabular}{|c|c|}
\hline \multicolumn{2}{|l|}{ Living condition } \\
\hline Living alone & $199(37.6 \%)$ \\
\hline Living with relatives & $250(47.3 \%)$ \\
\hline Institutionalized & $80(15.1 \%)$ \\
\hline \multicolumn{2}{|l|}{ Main medical diagnosis ${ }^{\mathrm{a}}$} \\
\hline Anemia & $380(71.8 \%)$ \\
\hline Infection/sepsis & $347(65.6 \%)$ \\
\hline Heart failure & $298(56.3 \%)$ \\
\hline Fall & $295(55.9 \%)$ \\
\hline Atrial fibrillation & $262(49.5 \%)$ \\
\hline Diabetes & $225(42.5 \%)$ \\
\hline Cancer & $210(39.7 \%)$ \\
\hline Obesity & $196(37.1 \%)$ \\
\hline Dementia & $185(35.0 \%)$ \\
\hline Kidney failure & $149(28.2 \%)$ \\
\hline Constipation & $137(25.9 \%)$ \\
\hline Chronic obstructive pulmonary disease & $115(21.7 \%)$ \\
\hline Urinary tract infection & $63(11.9 \%)$ \\
\hline Stroke & $58(11.0 \%)$ \\
\hline Liver failure & $40(7.6 \%)$ \\
\hline Body Mass Index $\left(\mathrm{kg} / \mathrm{m}^{2}\right)$ & $23.8 \pm 5.3$ \\
\hline \multicolumn{2}{|l|}{ Comprehensive Geriatric Assessment } \\
\hline Cumulative Illness Rating Scale & $4.6 \pm 2.0$ \\
\hline Exton Smith Scale & $15.6 \pm 3.7$ \\
\hline Basal Activities of Daily Living & $3.0 \pm 2.3$ \\
\hline Instrumental Activities of Daily Living & $3.6 \pm 3.1$ \\
\hline Short Portable Mental Status Questionnaire & $3.3 \pm 3.3$ \\
\hline Mini Nutritional Assessment-Short Form & $19.3 \pm 6.4$ \\
\hline Regularly used drugs $(n)$ & $5.7 \pm 3.1$ \\
\hline \multicolumn{2}{|l|}{ Hospital outcome } \\
\hline Discharged to home & $318(60.1 \%)$ \\
\hline Discharged to a healthcare facility & $201(38.0 \%)$ \\
\hline Transferred to other hospital department & $7(1.3 \%)$ \\
\hline Dead & $3(0.6 \%)$ \\
\hline
\end{tabular}

${ }^{a}$ Each individual patients may have more than one diagnosis

variables. The unadjusted MPI showed a moderate discrimination power (AUC 0.678 ; 95\% CI 0.628-0.729; $p<0.001$ ) at separating those patients who died from those who survived after 1 year since hospital admission. When adjusted for sex, age, GPS and/or serum osmolarity, the discrimination power became good in all explored models, with a progressive increase of AUC with the transition from Model 2 to Model 5. More in detail, when compared to Model 1 the addition of the above predictors increased the explained variance by $47.9 \%, 59.0 \%, 66.7 \%$ and $80.6 \%$ in Models 2, 3, 4 and 5 , respectively, while the discrimination power increased by $8.3 \%, 9.3 \%, 10.6 \%$ and $12.5 \%$ in the more complete predictive models, respectively (Table 3 ).
The best discrimination power in identifying patients who were at risk for 1-year mortality was demonstrated by Model 5 (AUC 0.763; 95\% CI 0.719-0.807; $p<0.001$ ). Figure 1 shows the ROCs for the unadjusted and fully adjusted logistic models. The bootstrap optimism estimate was 0.002; thus, the AUC corrected for optimism was 0.761 (95\% CI $0.724-0.798$ ), showing a good internal validity of the regression model.

The cumulative mortality rates were $11.2 \%(n=59)$, $23.4 \%(n=124)$ and $37.1 \%(n=196)$ after 60,180 and 365 days since hospital admission, respectively. The multivariate logistic regression analysis (Table 4) showed that severe risk MPI class $(>0.66)$ on admission was an independent predictor of mortality in all considered time intervals, while a similar predictive power was demonstrated for the moderate risk MPI class (0.33-0.66) only at 2 and 6 months. GPS-2 showed to be significantly associated with mortality at 180 and 365 days, with a trend toward an increased risk of death at 60-days (OR 3.393; $p=0.051$ ). Among the other considered covariates, male gender and age $>90$ years were predictive for both 180 and 365 days mortality, while at the latter time-point also age 85-90 years and current dehydration as measured by increased serum osmolarity resulted independently associated with mortality. The sensitivity analysis carried out via multivariate Cox regression models showed that MPI had a similar trend as an independent predictor of mortality in all considered time intervals, although the HRs were lower than the ORs found by fully adjusted logistic regression models (Fig. 2).

\section{Discussion}

In this study, we showed that-in addition to MPI moderate/high risk - severe inflammation as assessed by GPS-2 is independently associated with short/intermediate and long-term mortality in elderly patients acutely hospitalized, while demographic factors (age and gender) and hydration status are significant predictors only at longer time-points. In addition, the comprehensive model including severity of inflammation and frailty, age and sex significantly improves predictive accuracy of MPI alone.

The importance of inflammation and frailty in the management of older adults affected by acute diseases has been advocated by substantial literature [35, 36]. Accordingly, in this retrospective study we found a significant association between higher inflammation (as defined by GPS-2), severe frailty (MPI risk score $>0.66$ ) and greater intermediate- (6 months) and long-term mortality (1 year) in older patients acutely hospitalized.

Systemic inflammation is a feature of many acute conditions and is a strong predictor of adverse prognosis in adult and older patients $[15,37]$. The aging process is associated 
Table 2 Main characteristics of the study population and distribution according to 1-year mortality

\begin{tabular}{lllll}
\hline Variable & $\begin{array}{l}\text { All patients } \\
n=529\end{array}$ & $\begin{array}{l}\text { Survived } \\
n=333\end{array}$ & $\begin{array}{l}\text { Dead } \\
n=196\end{array}$ & $p$ value \\
\hline Age (years) & $84.6 \pm 7.3$ & $83.2 \pm 7.0$ & $86.9 \pm 7.1$ & $<0.001$ \\
$\leq 80$ & $147 ; 27.8$ & $111 ; 75.5 \%$ & $36 ; 24.5 \%$ & $<0.001$ \\
$81-85$ & $130 ; 24.6$ & $97 ; 74.6 \%$ & $33 ; 25.4 \%$ & \\
$86-90$ & $132 ; 25.0$ & $73 ; 55.3 \%$ & $59 ; 44.7 \%$ & \\
$>90$ & $120 ; 22.7$ & $52 ; 43.3 \%$ & $68 ; 56.7 \%$ & \\
Sex & & & & 0.029 \\
Female & $326 ; 61.6 \%$ & $217 ; 66.6 \%$ & $109 ; 33.4 \%$ & \\
Male & $203 ; 38.4 \%$ & $116 ; 57.1 \%$ & $87 ; 42.9 \%$ & $<0.001$ \\
Multidimensional Prognostic Index & $0.50 \pm 0.22$ & $0.44 \pm 0.20$ & $0.60 \pm 0.20$ & $<0.001$ \\
Low risk (0-0.33) & $150 ; 28.4$ & $119 ; 79.3 \%$ & $31 ; 20.7 \%$ & \\
Moderate risk (0.34-0.66) & $225 ; 42.5$ & $155 ; 68.9 \%$ & $70 ; 31.1 \%$ & \\
Severe risk (0.67-1) & $154 ; 29.1$ & $59 ; 38.3 \%$ & $95 ; 61.7 \%$ & \\
Glasgow Prognostic Score* & & & & \\
0 & $85 ; 17.1 \%$ & $69 ; 81.2 \%$ & $16 ; 18.8 \%$ & $<0.001$ \\
1 & $144 ; 17.2 \%$ & $103 ; 71.5 \%$ & $41 ; 28.5 \%$ & \\
2 & $252 ; 47.6 \%$ & $135 ; 53.6 \%$ & $117 ; 46.4 \%$ & \\
Serum osmolarity (mmol/L) & $295.1 \pm 13.6$ & $293.2 \pm 13.4$ & $298.4 \pm 13.3$ & $<0.001$ \\
$\leq 295$ & $274 ; 51.8$ & $193 ; 70.4 \%$ & $81 ; 29.6 \%$ & 0.001 \\
$296-300$ & $104 ; 19.7$ & $60 ; 57.7 \%$ & $44 ; 42.3 \%$ & \\
$>300$ & $151 ; 28.5$ & $80 ; 53.0 \%$ & $71 ; 47.0 \%$ & \\
\hline
\end{tabular}

Data are presented as: "mean \pm standard deviation" or "number; percentage". $*_{n} n=481$ to low-grade, systemic inflammation and dysregulation of many inflammatory pathways (the so called "inflammaging") [38]. These conditions are related to the occurrence of sarcopenia, insulin resistance, cardiovascular events and neurodegenerative disorders [39]. Moreover, systemic inflammation has been reported to play an important role in the prognosis of older patients [40,41], and increased levels of selected biomarkers, especially IL-6, have been shown to correlate with higher short-term mortality after hospitalization [16]. Here we extend these findings by showing that severity of inflammation measured at hospital admission is a good prognostic factor in the intermediate and long term, while in the short-term statistical significance was borderline $(p=0.051)$ possibly because of a significant number of missing data. In addition, our findings demonstrate that the Glasgow Prognostic Score is a valuable and clinically relevant parameter with prognostic implications. Previous studies have shown a strong association between albumin levels measured at admission and poor outcomes in older adults [42]. However, albumin is not considered a specific marker of inflammation as it also reflects nutritional status. The widespread use and applicability of more specific markers of inflammation is hampered by their availability in the clinical setting [16], therefore alternative biomarkers easily obtainable should be tested for predictivity. The Glasgow Prognostic Score, a simple tool based on routinely collected laboratory parameters, is easily computable and-by considering both serum albumin and CRP — might better reflect the impact of systemic inflammation on outcomes than one single variable. Available evidence on the shortterm mortality predictive power of CRP in geriatric patients acutely hospitalized is controversial $[15,16]$. Our results are consistent with the hypothesis that frailty is associated with higher inflammation, in line with previous studies reporting a correlation between increased serum levels of proinflammatory cytokines and frailty [36]. We believe that providing a model that incorporates GPS measured at hospital admission to the frailty score is of particular interest in terms of intermediate- and long-term risk stratification and management of frail older patients with acute conditions.

This study confirms the predictive reliability of MPI at all tested time-points, with the highest predictivity in the high-risk class. Since age and sex are biologically important factors in the association between mortality and frailty and the prognostic value of MPI becomes progressively higher when these variables are included in the model [14], we adjusted the predictivity analysis including these covariates. Our findings confirm an independent role for age and male sex on mortality as the impact of age increased progressively being maximal at 1 year for patients $>85$ years old. These results are consistent with previous studies showing that age and inflammation are associated in the development of age-related diseases and frailty [36, 43] and that mortality of older adults at any given frailty severity score 
Table 3 Logistic regression models to predict 1-year mortality

\begin{tabular}{|c|c|c|c|c|}
\hline Model & AUC $(95 \% \mathrm{CI}) ; p$ value & $R^{2} ; p$ value & Variables included in the final model & Adjusted $^{*}$ OR $(95 \% \mathrm{CI}) ; p$ value \\
\hline \multirow[t]{2}{*}{1} & \multirow[t]{2}{*}{$0.678(0.628-0.729) ;<0.001$} & \multirow[t]{2}{*}{$0.144 ;<0.001$} & MPI—moderate risk $(0.34-0.66)^{\dagger}$ & $1.734(1.067-2.818) ; 0.026$ \\
\hline & & & MPI—severe risk $(0.67-1.00)^{\dagger}$ & $6.181(3.706-10.310) ;<0.001$ \\
\hline \multirow[t]{4}{*}{2} & \multirow[t]{4}{*}{$0.734(0.688-0.781) ;<0.001$} & \multirow[t]{4}{*}{$0.213 ;<0.001$} & MPI-severe risk $(0.67-1.00)^{\dagger}$ & $5.213(2.997-9.069) ;<0.001$ \\
\hline & & & Age 86-90 years & 2.185 (1.266-3.772); 0.005 \\
\hline & & & Age $>90$ years & $2.814(1.578-5.016) ;<0.001$ \\
\hline & & & Sex $(\text { male })^{¥}$ & $2.081(1.384-3.128) ;<0.001$ \\
\hline \multirow[t]{5}{*}{3} & \multirow[t]{5}{*}{$0.741(0.695-0.788) ;<0.001$} & \multirow[t]{5}{*}{$0.229 ;<0.001$} & MPI—severe risk $(0.67-1.00)^{\dagger}$ & $4.980(2.847-8.709) ;<0.001$ \\
\hline & & & Age $86-90$ years $^{\ddagger}$ & $2.099(1.210-3.643) ; 0.008$ \\
\hline & & & Age $>90$ years ${ }^{\ddagger}$ & 2.775 (1.550-4.967); 0.001 \\
\hline & & & Sex $(\text { male })^{\ddagger}$ & 1.977 (1.310-2.984); 0.001 \\
\hline & & & Serum osmolarity $>300 \mathrm{mOsm} / \mathrm{L}^{\S}$ & $1.741(1.108-2.736) ; 0.016$ \\
\hline \multirow[t]{5}{*}{4} & \multirow[t]{5}{*}{$0.750(0.706-0.795) ;<0.001$} & \multirow[t]{5}{*}{$0.240 ;<0.001$} & MPI—severe risk $(0.67-1.00)^{\dagger}$ & $4.468(2.479-8.054) ;<0.001$ \\
\hline & & & Age 86-90 years ${ }^{\ddagger}$ & $2.000(1.127-3.551) ; 0.018$ \\
\hline & & & Age $>90$ years $^{\ddagger}$ & 2.700 (1.462-4.986); 0.002 \\
\hline & & & Sex $(\text { male })^{¥}$ & 1.822 (1.179-2.816); 0.007 \\
\hline & & & $\mathrm{GPS}=2^{\mathbb{I}}$ & $2.214(1.165-4.205) ; 0.015$ \\
\hline \multirow[t]{6}{*}{5} & \multirow[t]{6}{*}{$0.763(0.719-0.807) ;<0.001$} & \multirow[t]{6}{*}{$0.260 ;<0.001$} & MPI—severe risk $(0.67-1.00)^{\dagger}$ & $4.133(2.273-7.516) ;<0.001$ \\
\hline & & & Age $86-90$ years & $1.846(1.031-3.306) ; 0.039$ \\
\hline & & & Age $>90$ years $^{\ddagger}$ & 2.589 (1.394-4.807); 0.003 \\
\hline & & & Sex $(\text { male })^{\ddagger}$ & 1.705 (1.097-2.649); 0.018 \\
\hline & & & Serum osmolarity $>300 \mathrm{mOsm} / \mathrm{L}^{\S}$ & 2.069 (1.272-3.364); 0.003 \\
\hline & & & $\mathrm{GPS}=2^{\mathbb{I}}$ & 2.483 (1.289-4.780); 0.007 \\
\hline
\end{tabular}

$A U C$ area under receiver operating characteristic curve, $C I$ confidence interval, MPI Multidimensional Prognostic Index, GPS Glasgow Prognostic Score

*Except for model 1 (unadjusted OR)

${ }^{\dagger}$ Reference: MPI low risk (0.00-0.33)

Reference: age $\leq 80$ years

${ }^{¥}$ Reference: female sex

${ }^{\S}$ Reference: serum osmolarity $\leq 295 \mathrm{mOsm} / \mathrm{L}$

${ }^{\text {II }}$ Reference: $\mathrm{GPS}=0$ points

is sex-dependent, with male gender showing higher mortality risk than the female counterpart [11]. The association between male sex and mortality became significant at 6 months and 1 year, but not earlier, in line with a previous study which failed to demonstrate a male-female health survival paradox in hospitalized older adults for 28-day mortality [44]. The combined model including MPI and GPS corrected by age and sex had incremental prognostic value for mortality at 1 year compared with MPI alone. Compared to MPI alone (AUC 0.678), the AUC from multivariate regression models for predicting mortality increased when age and sex (AUC 0.734) and then GPS (AUC 0.750) were added (Table 2).

Another finding of interest is that impaired hydration was not associated with short- and intermediate-term mortality, but only with long-term mortality with an osmolarity cutoff $>300 \mathrm{mOsm} / \mathrm{L}$ indicating current dehydration. The lack of association between earlier mortality and dehydration can be explained by the clinical characteristics of the cohort, since only patients with mild acute diseases were admitted to the Geriatric Unit, while those with critical conditions including severe dehydration due to decompensated diabetes, acute kidney failure and water-loss hypernatremia were admitted to other hospital wards. Nonetheless, current dehydration was associated with long-term mortality and its addition to the comprehensive prognostic model increased its accuracy (AUC 0.763). Although a potential correlation between dehydration and specific domains of frailty including polypharmacy, functional decline, cognitive impairment and malnutrition has been suggested [45, 46], this link has not been fully demonstrated by available literature [23, 47]. The results of this study suggest that dehydration diagnosed at hospital admission is a predictive marker (although less robust than GPS, frailty and age) of long-term mortality and might therefore be taken into account when considering 1 -year mortality especially in high-risk patients. 


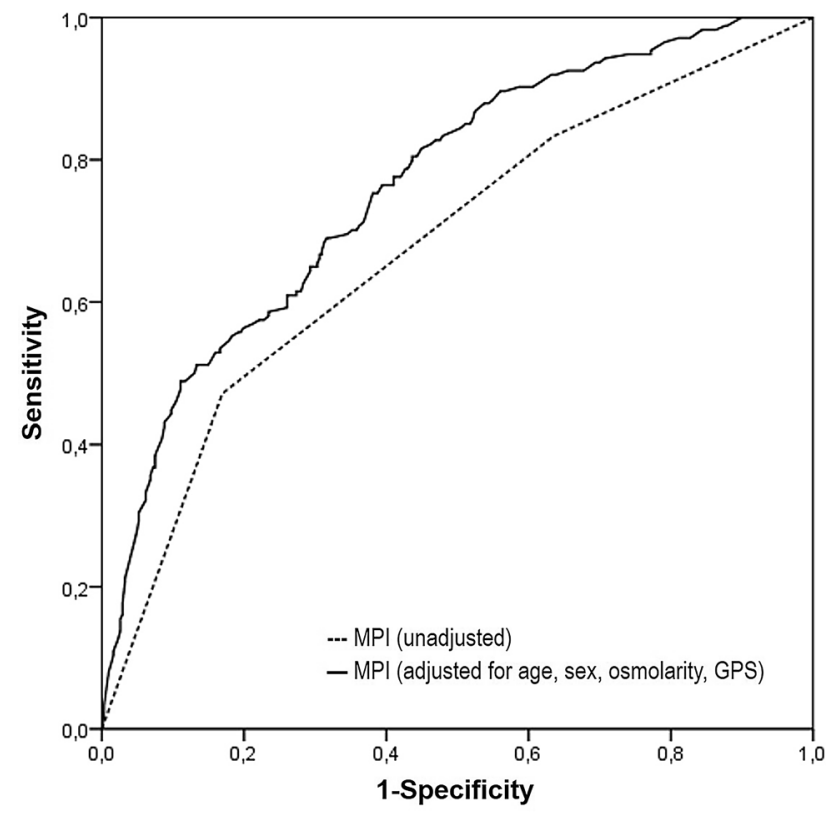

Fig. 1 Comparison of area under the curve (ROC) curves for unadjusted and adjusted multidimensional prognostic index (MPI)

Our findings might have important clinical consequences. All of the variables included in the model are easily obtainable and have proven to increase the long-term predictive accuracy of MPI. Inflammation and impaired hydration diagnosed at admission are potentially modifiable risk factors for severe outcomes and their management might provide important benefits for long-term survival. Unfortunately, interventional studies on the long-term effects of targeting inflammation and incident dehydration are missing. Future studies addressing these important issues are required.

The present investigation has strengths and limitations. A point of strength is that this study is one of the few assessing the role of inflammation, current dehydration and frailty on short- and long-term mortality in older adults. Since the confirmed internal validity of the final logistic regression model, out findings may be considered reliable in similar populations. However, our results should be considered in the light of some limitations. First the research was performed in a single Unit of a University Hospital, and the results may not be extended to patients treated in other hospitals or clinical units. Second, the database included patients with mild acute diseases, therefore the findings might not apply to patients with conditions at higher risk of lethality. Third, a shorter mortality endpoint (e.g., 30 days) was not considered in the analyses due to an insufficient number of death events in this time-span, precluding to appropriately build the multivariable regression models. Therefore, our results should be confirmed by replicating the study across different populations and with larger samples.

In conclusion, inflammation as scored by GPS-2 and MPI-severe risk were predictive for a higher risk of mortality in the short to long term in acutely hospitalized older patients. Current dehydration, male sex and age $>85$ years were associated with increased risk of mortality only in the mid and/or long term. Since demographic, inflammation and hydration biomarkers are not included in the MPI-which in this way may not represent completely enough the risk of death - the inclusion of these variables might increase the prognostic accuracy of the MPI in clinical practice when assessed on hospital admission. Diagnosing and targeting

Table 4 Stepwise multiple logistic regression analysis of mortality at different time interval from hospital admission on study variables

\begin{tabular}{|c|c|c|c|}
\hline & $\begin{array}{l}\text { 60-day mortality } \\
\text { OR }(95 \% \mathrm{CI}) ; p \text { value }\end{array}$ & $\begin{array}{l}\text { 180-day mortality } \\
\text { OR }(95 \% \mathrm{CI}) ; p \text { value }\end{array}$ & $\begin{array}{l}\text { 365-day mortality } \\
\text { OR }(95 \% \mathrm{CI}) ; p \text { value }\end{array}$ \\
\hline MPI—low risk & Reference & Reference & Reference \\
\hline MPI—moderate risk & 4.309 (1.237-15.009); 0.022 & $2.133(1.062-4.286) ; 0.033$ & $n . s$ \\
\hline MPI—severe risk & $11.464(3.373-38.958) ;<0.001$ & $5.408(2.632-11.111) ;<0.001$ & $4.133(2.273-7.516) ;<0.001$ \\
\hline $\mathrm{GPS}=0$ & Reference & Reference & Reference \\
\hline $\mathrm{GPS}=1$ & $n . s$ & $n . s$ & $n . s$ \\
\hline $\mathrm{GPS}=2$ & 3.393 (0.992-11.602); 0.051 & 3.010 (1.275-7.107); 0.012 & 2.483 (1.289-4.780); 0.007 \\
\hline Sex (male) & $n . s$ & 1.809 (1.109-2.950); 0.017 & 1.705 (1.097-2.649); 0.018 \\
\hline Age $(\leq 80$ years $)$ & $n . s$ & Reference & Reference \\
\hline $81-85$ years & n.s & $n . s$ & $n . s$ \\
\hline $86-90$ years & $n . s$ & $n . s$ & $1.846(1.031-3.306) ; 0.039$ \\
\hline$>90$ years & $n . s$ & $2.470(1.250-4.881) ; 0.009$ & 2.589 (1.394-4.807); 0.003 \\
\hline Euhydration & $n . s$ & $n . s$ & Reference \\
\hline Impending dehydration & $n . s$ & $n . s$ & $n . s$ \\
\hline Current dehydration & n.s & $n . s$ & $2.069(1.272-3.364) ; 0.003$ \\
\hline
\end{tabular}

OR odds ratio, CI confidence interval, MPI Multidimensional Prognostic Index, GPS Glasgow Prognostic Score, n.s. nonsignificant 

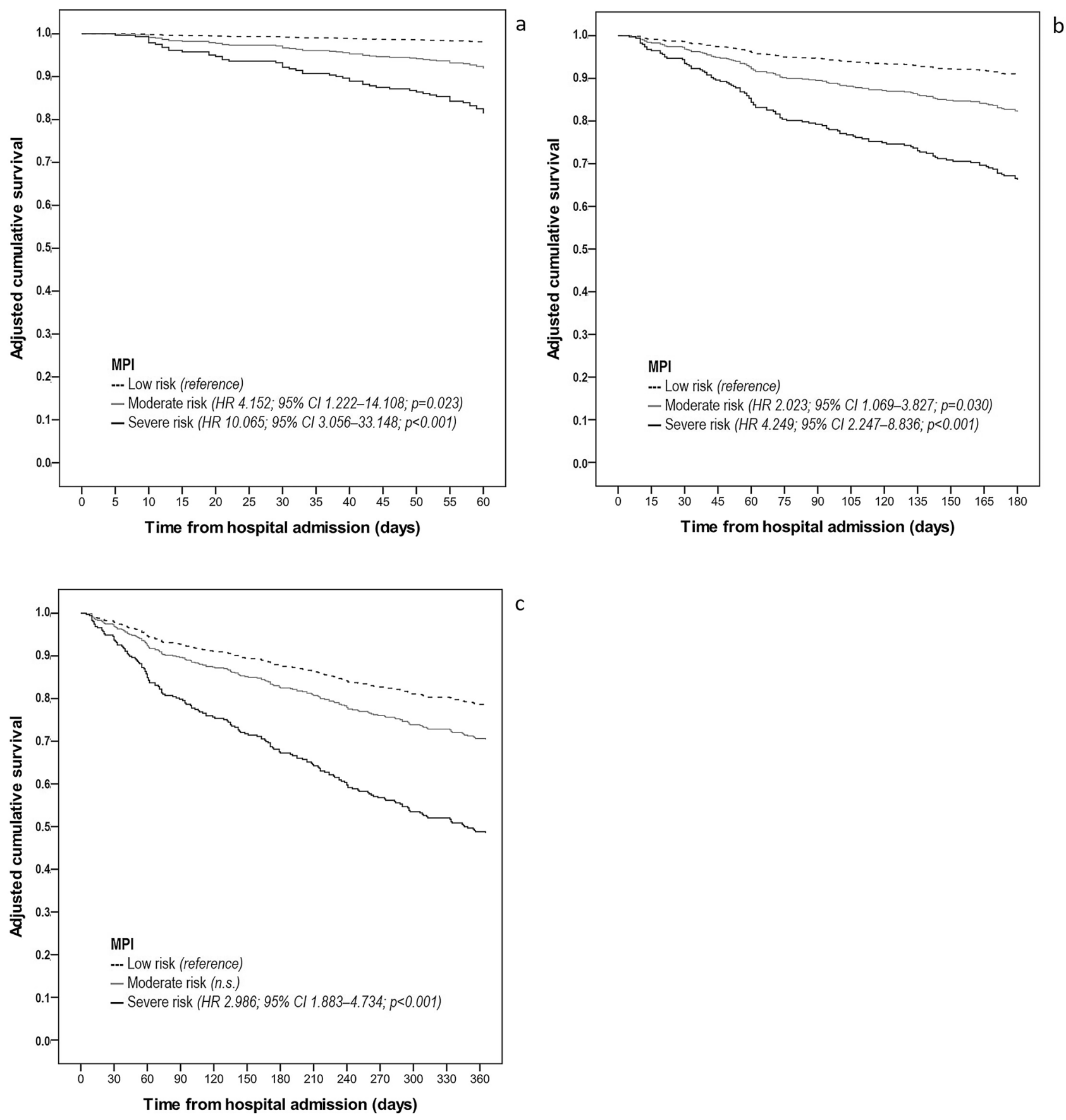

c

Fig. 2 Adjusted survival curve (multivariate Cox regression analysis) at 60 days (a), 180 days (b) and 365 days (c) from hospital admission for patients belonging to different multidimensional prognostic index (MPI) categories

inflammation and dehydration both in hospital and after discharge might carry beneficial effects for early- and longterm survival. Further studies are needed to confirm this hypothesis.

Acknowledgements We gratefully acknowledge the cooperation of the patients, who made this work possible.
Funding Open access funding provided by Università degli Studi di Trieste within the CRUI-CARE Agreement. This research did not receive any specific grant from funding agencies in the public, commercial, or not-for-profit sectors.

Availability of data and materials On request.

Code availability Not applicable. 


\section{Declarations}

Conflicts of interest The authors declare they have no conflict of interest.

Ethics approval See "Materials and methods" section.

Consent to participate See "Materials and methods" section.

Open Access This article is licensed under a Creative Commons Attribution 4.0 International License, which permits use, sharing, adaptation, distribution and reproduction in any medium or format, as long as you give appropriate credit to the original author(s) and the source, provide a link to the Creative Commons licence, and indicate if changes were made. The images or other third party material in this article are included in the article's Creative Commons licence, unless indicated otherwise in a credit line to the material. If material is not included in the article's Creative Commons licence and your intended use is not permitted by statutory regulation or exceeds the permitted use, you will need to obtain permission directly from the copyright holder. To view a copy of this licence, visit http://creativecommons.org/licenses/by/4.0/.

\section{References}

1. Boult C, Dowd B, McCaffrey D et al (1993) Screening elders for risk of hospital admission. J Am Geriatr Soc 41:811-817. https:// doi.org/10.1111/j.1532-5415.1993.tb06175.x

2. Hubbard RE, Peel NM, Samanta M et al (2017) Frailty status at admission to hospital predicts multiple adverse outcomes. Age Ageing 46:801-806. https://doi.org/10.1093/ageing/afx081

3. Khatry K, Peel NM, Gray LC et al (2018) The utility of the frailty index in clinical decision making. J Frailty Aging 7:138-141. https://doi.org/10.14283/jfa.2018.7

4. Cruz-Jentoft AJ, Daragjati J, Fratiglioni L et al (2020) Using the Multidimensional Prognostic Index (MPI) to improve costeffectiveness of interventions in multimorbid frail older persons: results and final recommendations from the MPI_AGE European Project. Aging Clin Exp Res 32:861-868. https://doi.org/10.1007/ s40520-020-01516-0

5. Pilotto A, Custodero C, Maggi S et al (2020) A multidimensional approach to frailty in older people. Ageing Res Rev 60:101047. https://doi.org/10.1016/j.arr.2020.101047

6. Warnier RM, van Rossum E, van Velthuijsen E et al (2016) Validity, reliability and feasibility of tools to identify frail older patients in inpatient hospital care: a systematic review. J Nutr Health Aging 20:218-230. https://doi.org/10.1007/s12603-015-0567-z

7. Argano C, Scichilone N, Natoli G et al (2021) Pattern of comorbidities and 1-year mortality in elderly patients with COPD hospitalized in internal medicine wards: data from the RePoSI Registry. Intern Emerg Med 16:389-400. https://doi.org/10.1007/ s11739-020-02412-1

8. Golüke NMS, van de Vorst IE, Vaartjes IH et al (2019) Risk factors for in-hospital mortality in patients with dementia. Maturitas 129:57-61. https://doi.org/10.1016/j.maturitas.2019.08.007

9. Tai S, Li X, Yang H et al (2020) Sex differences in the outcomes of elderly patients with acute coronary syndrome. Cardiol Res Pract 2020:5091490. https://doi.org/10.1155/2020/5091490

10. Walicka M, Chlebus M, Śliwczyński A et al (2020) Predictors of in-hospital mortality in nonsurgical departments: a multivariable regression analysis of 2855029 hospitalizations. Polish Arch Intern Med 130:268-275. https://doi.org/10.20452/Pamw.15185
11. Gordon EH, Peel NM, Samanta M et al (2017) Sex differences in frailty: a systematic review and meta-analysis. Exp Gerontol 89:30-40. https://doi.org/10.1016/j.exger.2016.12.021

12. Hubbard RE, Peel NM, Samanta M et al (2015) Derivation of a frailty index from the interRAI acute care instrument. BMC Geriatr 15:27. https://doi.org/10.1186/s12877-015-0026-z

13. Pilotto A, Veronese N, Siri G et al (2020) Association between the multidimensional prognostic index and mortality over 15 years of follow-up in the Inchianti study. J Gerontol Ser A Biol Sci Med Sci. https://doi.org/10.1093/gerona/glaa237

14. Bryant K, Sorich MJ, Woodman RJ et al (2019) Validation and adaptation of the multidimensional prognostic index in an older australian cohort. J Clin Med 8:1820. https://doi.org/10.3390/ jcm8111820

15. Ticinesi A, Lauretani F, Nouvenne A et al (2017) C-reactive protein (CRP) measurement in geriatric patients hospitalized for acute infection. Eur J Intern Med 37:7-12. https://doi.org/10. 1016/j.ejim.2016.08.026

16. Rizza S, Morabito P, De Meo L et al (2021) IL-6 levels influence 3-month all-cause mortality in frail hospitalized older patients. Aging Dis 12:353-359. https://doi.org/10.14336/ad.2020.0713

17. Forrest LM, McMillan DC, McArdle CS et al (2004) Comparison of an inflammation-based prognostic score (GPS) with performance status (ECOG) in patients receiving platinum-based chemotherapy for inoperable non-small-cell lung cancer. Br J Cancer 90:1704-1706. https://doi.org/10.1038/sj.bjc.6601789

18. Kang HS, Cho KW, Kwon SS et al (2018) Prognostic significance of Glasgow prognostic score in patients with acute exacerbation of idiopathic pulmonary fibrosis. Respirology (Carlton, Vic) 23:206-212. https://doi.org/10.1111/resp.13184

19. Kuluöztürk M, Deveci F (2020) The Glasgow prognostic score can be a predictor of mortality in acute exacerbation of chronic obstructive pulmonary disease. Expert Rev Respir Med 14:521525. https://doi.org/10.1080/17476348.2020.1735366

20. Wang R, Wen X, Huang C et al (2019) Association between inflammation-based prognostic scores and in-hospital outcomes in elderly patients with acute myocardial infarction. Clin Interv Aging 14:1199-1206. https://doi.org/10.2147/cia.S214222

21. Altay S, Gürdoğan M, Keskin M et al (2019) The inflammation-based glasgow prognostic score as a prognostic factor in patients with intensive cardiovascular care unit. Medicina (Kaunas) 55:139. https://doi.org/10.3390/medicina55050139

22. Sanson G, Marzinotto I, De Matteis D et al (2020) Impaired hydration status in acutely admitted older patients: prevalence and impact on mortality. Age Ageing. https://doi.org/10.1093/ ageing/afaa264

23. Wojszel ZB (2020) Impending low intake dehydration at admission to a geriatric ward- prevalence and correlates in a crosssectional study. Nutrients 12:398. https://doi.org/10.3390/nu120 20398

24. Hooper L, Abdelhamid A, Attreed NJ et al (2015) Clinical symptoms, signs and tests for identification of impending and current water-loss dehydration in older people. Cochrane Database Syst Rev 2015:cd009647. https://doi.org/10.1002/14651 858.CD009647.pub2

25. Volkert D, Beck AM, Cederholm T et al (2019) ESPEN guideline on clinical nutrition and hydration in geriatrics. Clin Nutr (Edinburgh, Scotland) 38:10-47

26. Dharmarajan K, Swami S, Gou RY et al (2017) Pathway from delirium to death: potential in-hospital mediators of excess mortality. J Am Geriatr Soc 65:1026-1033. https://doi.org/10.1111/ jgs. 14743

27. El-Sharkawy AM, Devonald MAJ, Humes DJ et al (2020) Hyperosmolar dehydration: a predictor of kidney injury and outcome in hospitalised older adults. Clin Nutr (Edinburgh, 
Scotland) 39:2593-2599. https://doi.org/10.1016/j.clnu.2019. 11.030

28. Manabe T, Teramoto S, Tamiya $\mathrm{N}$ et al (2015) Risk factors for aspiration pneumonia in older adults. PLoS ONE 10:e0140060. https://doi.org/10.1371/journal.pone.0140060

29. Pilotto A, Ferrucci L, Franceschi M et al (2008) Development and validation of a multidimensional prognostic index for one-year mortality from comprehensive geriatric assessment in hospitalized older patients. Rejuvenation Res 11:151-161. https://doi.org/10. 1089/rej.2007.0569

30. Khajuria A, Krahn J (2005) Osmolality revisited—deriving and validating the best formula for calculated osmolality. Clin Biochem 38:514-519. https://doi.org/10.1016/j.clinbiochem.2005.03. 001

31. Hooper L, Abdelhamid A, Ali A et al (2015) Diagnostic accuracy of calculated serum osmolarity to predict dehydration in older people: adding value to pathology laboratory reports. BMJ Open 5:e008846. https://doi.org/10.1136/bmjopen-2015-008846

32. Hanley JA, McNeil BJ (1982) The meaning and use of the area under a receiver operating characteristic (ROC) curve. Radiology 143:29-36. https://doi.org/10.1148/radiology.143.1.7063747

33. Steyerberg EW, Harrell FE Jr, Borsboom GJ et al (2001) Internal validation of predictive models: efficiency of some procedures for logistic regression analysis. J Clin Epidemiol 54:774-781. https:// doi.org/10.1016/s0895-4356(01)00341-9

34. Skalská H, Freylich VJ (2006) Web-bootstrap estimate of area under ROC curve. Aust J Stat 35:325-330

35. Marcos-Pérez D, Sánchez-Flores M, Proietti S et al (2020) Association of inflammatory mediators with frailty status in older adults: results from a systematic review and meta-analysis. GeroScience 42:1451-1473. https://doi.org/10.1007/s11357-020-00247-4

36. Soysal P, Stubbs B, Lucato P et al (2016) Inflammation and frailty in the elderly: a systematic review and meta-analysis. Ageing Res Rev 31:1-8. https://doi.org/10.1016/j.arr.2016.08.006

37. Nouvenne A, Ticinesi A, Lauretani F et al (2016) The prognostic value of high-sensitivity $\mathrm{C}$-reactive protein and prealbumin for short-term mortality in acutely hospitalized multimorbid elderly patients: a prospective cohort study. J Nutr Health Aging 20:462468. https://doi.org/10.1007/s12603-015-0626-5
38. Xia S, Zhang X, Zheng S et al (2016) An update on inflammaging: mechanisms, prevention, and treatment. J Immunol Res 2016:8426874. https://doi.org/10.1155/2016/8426874

39. Michaud M, Balardy L, Moulis G et al (2013) Proinflammatory cytokines, aging, and age-related diseases. J Am Med Dir Assoc 14:877-882. https://doi.org/10.1016/j.jamda.2013.05.009

40. Sanson G, Sadiraj M, Barbin I et al (2020) Prediction of early- and long-term mortality in adult patients acutely admitted to internal medicine: NRS-2002 and beyond. Clin Nutr (Edinburgh, Scotland) 39:1092-1100. https://doi.org/10.1016/j.clnu.2019.04.011

41. Wei L, Xie H, Yan P (2020) Prognostic value of the systemic inflammation response index in human malignancy: a meta-analysis. Medicine 99:e23486. https://doi.org/10.1097/md.0000000000 023486

42. Cabrerizo S, Cuadras D, Gomez-Busto F et al (2015) Serum albumin and health in older people: review and meta analysis. Maturitas 81:17-27. https://doi.org/10.1016/j.maturitas.2015.02.009

43. Fulop T, Witkowski JM, Olivieri F et al (2018) The integration of inflammaging in age-related diseases. Semin Immunol 40:17-35. https://doi.org/10.1016/j.smim.2018.09.003

44. Gordon EH, Peel NM, Hubbard RE (2018) The male-female health-survival paradox in hospitalised older adults. Maturitas 107:13-18. https://doi.org/10.1016/j.maturitas.2017.09.011

45. Stookey JD, Purser JL, Pieper CF et al (2004) Plasma hypertonicity: another marker of frailty? J Am Geriatr Soc 52:1313-1320. https://doi.org/10.1111/j.1532-5415.2004.52361.x

46. McCrow J, Morton M, Travers C et al (2016) Associations between dehydration, cognitive impairment, and frailty in older hospitalized patients: an exploratory study. J Gerontol Nurs 42:19-27. https://doi.org/10.3928/00989134-20160201-01

47. Hooper L, Bunn DK, Downing A et al (2016) Which frail older people are dehydrated? The UK DRIE study. J Gerontol A Biol Sci Med Sci 71:1341-1347. https://doi.org/10.1093/gerona/glv205

Publisher's Note Springer Nature remains neutral with regard to jurisdictional claims in published maps and institutional affiliations. 